\title{
Closed-loop Control System Based on the Trajectory Information
}

\author{
Huaiyuan Wang, Baohui Zhang \\ Songhao Yang, Hao Yang \\ State key laboratory of electrical insulation and power \\ equipment \\ Xi'an Jiaotong University \\ Xian, 710049,China \\ wanghuaiy@stu.xjtu.edu.cn
}

\author{
Lihua Wu, Qing Wang, Xiaojun Li \\ Shiying Ma, Junjie Lin \\ China Electric Power Research Institute \\ Beijing 100192, China
}

\begin{abstract}
Based on trajectory information for the electric power system, this paper analyzes Transient Stability Prediction and new generation transient stability control of large power system. This method, which is independent of system parameter and model, can tell the transient stability of the electric power system faster and give the control strategy by the closed-loop control system. Firstly, System instability is detected by the characteristics concave or convex of the critical machine trajectory of the post-fault system. Secondly, to accelerate the speed of recognize the transient stability of the power system, the self-memory numerical predictive formula of multi-variables is derived. Thirdly, the control strategy by the closed-loop control system is given based on transient kinetic energy, when the power system is unstable.
\end{abstract}

Keywords-Electric power system; characteristic concave or convex of surface; Transient instability prediction; Closed-loop control system.

\section{INTRODUCTION}

With the rapid development of the modern society, the scale of the electric power system is becoming larger and larger. Following a large disturbance, a power system will recover to a stable state if various effective measures can be taken in time. But, some of the emergency measures like generator tripping and controlled system separation should be exercised only when there is an absolute necessity. Therefore, a fast and accurate method of distinguishing between stable and unstable post-fault trajectory is necessary. When discovering that the electric power system is unstable, an effective measure is inevitable.

This paper proposes a closed-loop control system using state-plane plot of speed and power angle to detect the system instability and transient kinetic energy to obtain the control strategy. In this paper, the instability detection method [1][2] cooperating with self-memory numerical predictive theory [3], detect the system instability. And then, the control strategy is given based on transient kinetic energy, when the power system is unstable. The simulation studies show that the proposed closed-loop control system [4] is fast, computationally efficient, and realizable.

\section{POWER System InStabiLITy Detection}

A. Identification theory of transient instability for autonomous SMIB

For the SMIB system, the equations of electromechanical transient process are described as

$$
\begin{aligned}
& \dot{\delta}=\omega_{0} \Delta \omega \\
& \dot{M} \Delta \omega=P_{M}-P_{e m i} \sin \delta-D \omega_{0} \Delta \omega=f(\delta, \Delta \omega)
\end{aligned}
$$

In stability analysis, second derivative of $\Delta \omega$ means trajectory's geometric feature, which can be described as $l=\frac{d^{2} \Delta \omega}{d \delta^{2}}$.

The necessary and sufficient condition of transient instability of power system can be obtained: $l \cdot \Delta \omega>0$, and the discrete form can be written as:

Where,

$$
\tau=k(i)-k(i-1)>0
$$

$$
k(i)=\frac{\Delta \omega(i)-\Delta \omega(i-1)}{\delta(i)-\delta(i-1)}
$$

The conclusion is proved in [1][2].If the index $\tau$ is always less than zero, the system will be stable, and the system will lose synchronization if $\tau$ is larger than zero immediately or sustain a short time after the fault-clearing time.

B. Identification theory of transient instability for nonautonomous SMIB system

The equations of electromechanical transient process for non-autonomous SMIB system can be described as follows:

$$
\begin{aligned}
& \frac{d \delta}{d t}=\omega_{0} \Delta \omega \\
& M \frac{d \Delta \omega}{d t}=P_{M}(t)-P_{e}(t, \delta)=\Delta P(t, \delta)
\end{aligned}
$$

Where the right function of motion equation involves the variable of time, and thus $\Delta P(t, \delta)$ doesn't represent a strict 
sine curve any more. As a consequence, the index $\tau$ greater than zero in a certain time can only indicated the instability of the autonomous system in that parameter condition, but not the non-autonomous system.

$$
\begin{aligned}
& P_{e}\left(t_{i}, \delta\right)=\lambda_{0}\left(t_{i}\right)-\lambda_{1}\left(t_{i}\right) \cos \delta-\lambda_{2}\left(t_{i}\right) \sin \delta \\
& \Delta P\left(t_{i}, \delta\right)=P_{c}\left(t_{i}\right)-\lambda_{1}\left(t_{i}\right) \cos \delta-\lambda_{2}\left(t_{i}\right) \sin \delta
\end{aligned}
$$

To fortify the accuracy of the detection, append two indexes. One is the feature index $\mu$ of trajectory in stateplane of unbalanced power and power angle, and the other is the assessment $\varepsilon \cdot \Delta \omega$ of the impact of the parameters timevarying on unbalanced power [5].

The feature index $\mu$ of trajectory in state-plane of unbalanced power and power angle is defined as

Where,

$$
\mu=\Delta P^{\prime}(i)-\Delta P^{\prime}(i-1)>0
$$

$$
\Delta P^{\prime}(i)=\frac{\Delta P(i)-\Delta P(i-1)}{\delta(i)-\delta(i-1)}
$$

Suppose that unbalanced power can be measured, the values of time-varying parameters $P_{c}(t), \lambda_{1}(t)$ and $\lambda_{2}(t)$ can be identified with least square methodbased on the measured trajectory.

$$
Y=\left[\begin{array}{c}
P_{c} \\
\lambda_{1} \\
\lambda_{2}
\end{array}\right]=\left(A^{T} A\right)^{-1} A^{T} b
$$

Since unbalanced power is impacted both by $Y$ and $\delta$, to assess only the impact of $Y$, we should fix $\delta\left(t_{i}\right)=\beta$. Thus, (4) can be written

$$
\Delta P_{\beta}(Y(t))=P_{c}(t)-\lambda_{1}(t) \cos \beta-\lambda_{2}(t) \sin \beta
$$

At the moment $t_{i-1}$, the unbalanced power with timevarying parameter $Y\left(t_{i-1}\right)$ and current $\delta$ is

$$
\Delta P_{\beta}\left(Y\left(t_{i-1}\right)\right)=\Delta P\left(Y\left(t_{i-1}\right), \beta\right)
$$

Then the assessment of the impact of the parameters time-varying on unbalanced power is defined as below:

$$
\varepsilon=\Delta P_{\beta}\left(Y\left(t_{i}\right)\right)-\Delta P_{\beta}\left(Y\left(t_{i-1}\right)\right)
$$

Eventually, instability criterion for non-autonomous SMIB system can be described as:

$$
\tau>0 \& \mu>0 \& \varepsilon \cdot \Delta \omega>0
$$

\section{SMIB EQUIVALENT}

\section{A. Divide the power system into two groups}

Though the generator swing curves in real time, identify the absolute dominant mode to divide the power system into two groups. Calculating the gaps between the adjacent generator swing curves, choose the first biggest gap $\theta_{1}$ and the second one $\theta_{2}$.When the gaps satisfy the follow equation, the first biggest gap is the absolute dominant mode.

$$
\theta_{1} \geq k \theta_{2}
$$

Where, the $\mathrm{k}$ may choose $2 \sim 5$. Therefore, the power system is reduced to a two-machine system. For transient stability studies, equations for the power system with $\mathrm{m}$ buses, $\mathrm{n}$ generators and other dynamic devices corresponding to partial center of inertia can be described by detailed compact model as follow [6] [7]:

$$
\begin{array}{ll}
\delta_{\text {PCOI }}=\frac{1}{M_{T}} \sum_{i=1}^{n} M_{i} \delta_{i} \quad \omega_{\text {PCOI }}=\frac{1}{M_{T}} \sum_{i=1}^{n} M_{i} \omega_{i} \\
M_{T}=\sum_{i=1}^{n} M_{i}
\end{array}
$$

Where, $M_{i}$ is inertia constant of machine i under synchronous frame model. $\delta_{i}$ and $\omega_{i}$ are generator angle and angular velocity of machine $\mathrm{i}$ with respect to a synchronous frame.

The two-machine system can be described as follow:

$$
\left\{\begin{array}{l}
M_{s} \ddot{\delta}_{s}=P_{m s}-P_{e s} \\
M_{a} \ddot{\delta}_{a}=P_{m a}-P_{e a}
\end{array}\right.
$$

Where, $\delta_{a}, \delta_{s}$ are the partial center of angles of the generators in area $\mathrm{A}$ and $\mathrm{S}$, respectively. $M_{a}, M_{S}$ are the sum of the inertia constant of the generators in area A and S, respectively.

\section{B. SMIB equivalent}

The two-machine system can be reduced to a SMIB equivalent system, and the equation can be described as follow:

Where,

$$
M \ddot{\delta}=P_{m}-P_{e}
$$

$$
\begin{gathered}
\delta=\delta_{s}-\delta_{a} \\
M=\frac{M_{a} M_{s}}{M_{a}+M_{S}} \\
P_{m}=\left(M_{a} \sum_{i \in S} P_{m i}-M_{s} \sum_{j \in A} P_{m j}\right) / M_{T} \\
P_{e}=\left(M_{a} \sum_{i \in S} P_{e i}-M_{S} \sum_{j \in A} P_{e j}\right) / M_{T}
\end{gathered}
$$

$M_{T}$ is the sum of the inertia constant of the generators in the whole system. $P_{m}$ and $P_{e}$ are the mechanical input power and electrical output power of SMIB equivalent, respectively.

\section{SELF-MEMORY NUMERICAL PREDICTIVE Formula}

Generally, when the detection technique identify that the system is instability, emergent control is difficult to be executed in time. To accelerate the detection of the system instability, predictive formula can be employed based on the generator swing curves in real time. Self-memory numerical predictive formula is employed in this paper, and the function of the third order can be described as follow: 


$$
\begin{gathered}
\delta_{i+1}=\beta_{-2}\left(\delta_{i-2}-\delta_{i-1}+2 \omega_{i-2} \Delta t\right)+ \\
\beta_{-1}\left(\delta_{i-2}-\delta_{i}+2 \omega_{i-1} \Delta t\right)+\beta_{0}\left(\delta_{i-1}+2 \omega_{i} \Delta t\right)+\delta_{i} \beta_{1} \\
\Delta P_{i+1}=P_{C}(t)+\lambda_{1 t}(t) \sin \left(\delta_{i+1}\right)+\lambda_{2 t}(t) \cos \left(\delta_{i+1}\right) \\
\Delta \omega_{i+1}=\Delta \omega_{i}+\frac{1}{2 M}\left[\Delta P_{i}+\Delta P_{i+1}\right] \cdot \Delta t
\end{gathered}
$$

The procedure of the self-memory numerical predictive formula is described in [3]. Firstly, based on the current data including the generator angle and angular velocity of SMIB equivalent, predict the next moment generator angle $\delta_{i+1}$ employing formula (11). Secondly, based on generator angle $\delta_{i+1}$ and the least square method, the future unbalanced power $\Delta P_{i+1}$ can be obtained. Thirdly, according to the unbalanced power $\Delta P_{i}$ and $\Delta P_{i+1}$, the next angular velocity $\Delta \omega_{i+1}$ can be calculated by formula (13). Thus, the next moment data can be obtained by rolling prediction employing the self-memory numerical predictive formula above.

\section{Control STRATEgy}

\section{A. The computation of the generator tripping value}

As a common sense, the system out of synchronism means that the angular velocity of the generators is different from each other. Thus, if the angular velocity of the generators can be synchronous by employing control strategy, the power system will recover to a stable state. Suppose that the difference between the angular velocity of the generators is the redundant transient kinetic energy which contribute to the instability, the system can recover stability by employing control strategy to remove the redundant energy. Through a mass of simulation, draw the conclusion that the system will recover stability when the transient kinetic energy between center of inertia of the system and critical group of the generators is removed.

Suppose that the system is identified instability at $T_{e}$ by the generator swing curves, the transient kinetic energy between center of inertia of the system and the generators in area $\mathrm{S}$ at $T_{e}$ is defined as redundant transient kinetic energy. The redundant energy can be described as:

$$
E_{K, e}=\frac{1}{2} M_{S}\left(\omega_{S, e}-\omega_{C O I, e}\right)^{2}
$$

Where, $E_{K, e}$ is the redundant energy at $T_{e} \cdot M_{S}$ is the sum of the inertia constant of the generators in area S. $\omega_{s, e}$ and $\omega_{\mathrm{CO}, e}$ are the center angular velocity of inertia of the critical generators and whole system at $T_{e}$, respectively.

\section{B. The choice of the control location}

To choose the control location, the index $\varpi_{i, e}$ is defined as:

$$
\varpi_{i, e}=\tilde{\theta}_{i, e} \cdot \tilde{\omega}_{i, e}
$$

Where, $\tilde{\theta}_{i, e}$ and $\tilde{\omega}_{i, e}$ are generator angle and angular velocity of machine $i$ with respect to a synchronous frame at $T_{e}$. When $\varpi_{i, e}$ is greater than zero, the system is likely to be instability. Thus, the control location is chosen based on the index $\varpi_{i, e}$ rank ordering. Select the generators that the index $\varpi_{i, e}>0$ from large to small to compose a control location set.

The transient kinetic energy between the generator $\mathrm{i}$ in area $\mathrm{S}$ and the center of inertia of the generators in area $\mathrm{A}$ is defined as the effect to the redundant energy in shutting off this generator.

$$
E_{K i, e}=\frac{1}{2} M_{i}\left(\omega_{i, e}-\omega_{a, e}\right)^{2}
$$

Where, $\omega_{i, e}$ is the angular velocity of machine $\mathrm{i}$ in area S. $\omega_{a, e}$ is the center angular velocity of inertia of the generators in area $\mathrm{A}$.

When the generator tripping value $E_{K, e}$ is calculated, choose the generators that and the sum of $E_{K i, e}$ is greater than and nearest to $E_{K, e}$ in the control location set based on index $\varpi_{i, e}$ rank ordering.

\section{Simulation RESUlt}

To verify the proposed method, simulations are performed using the computer simulation of 36 buses system with 8 generators (EPRI-36 China). The power system model is as follows: a classical generator model with constant mechanical power, and linear load and network models. The single-line diagram is shown in Fig 1.

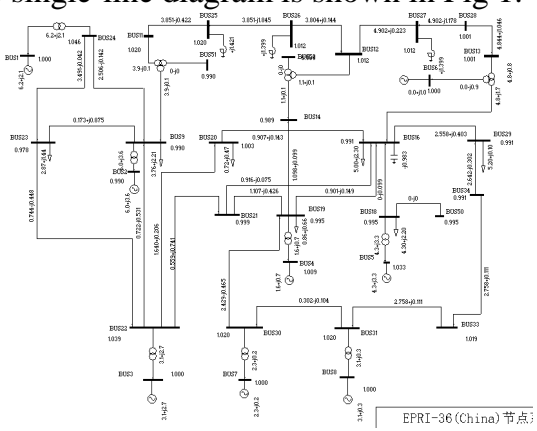

Fig 1. Single-line diagram of the EPRI-36 (China)

Three-phase short-circuits happens on bus-33 and the during-fault time interval of the disturbance is $0.24 \mathrm{~s}$. The generators swing curves are shown in Fig.2, and the critical 
group contains G7 and G8.

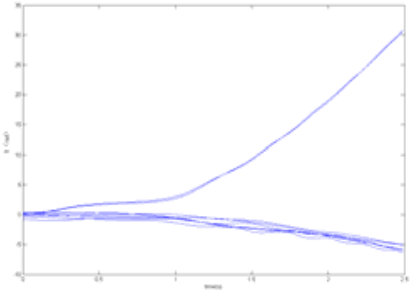

Fig 2. The swing curves of the generators without control

When the disturbance is eliminated, predictive technique is activated. The indexes are calculated based on the data predicted by the technique, which are shown in Fig.3.
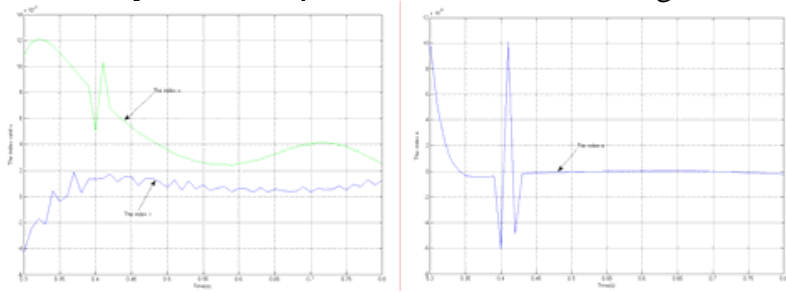

Fig 3. The calculated indexes curves

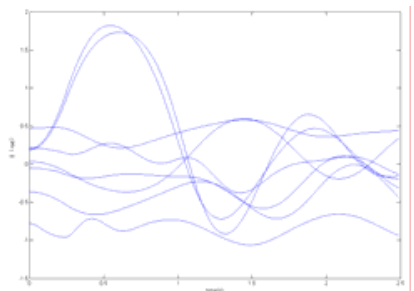

Fig 4. The swing curves of the generators after control

As is shown in Fig.3, the indexes are greater than zero at $0.41 \mathrm{~s}$ simultaneously based on the data predicted by the technique. Thus, the time when detective the instability is $0.3 \mathrm{~s}$, and the control strategy is started. Though formula (14), the redundant energy is 1.0 (p.u). G7 is chosen by formula (15) that G7 is 1.0 (p.u.) and G8 is 0.4 (p.u). Then calculated the $E_{K 7}$ by formula (16), and the result is 1.7(p.u). Thus, the control strategy is obtained that shut off $60 \%$ of G7. Suppose that the strategy is employed at $0.4 \mathrm{~s}$ and the swing curves after control are shown in Fig.4. The system recovers to a stable state after the control.

\section{CONCLUSION}

In conclusion, the detective technique can identify the instability accurately and quickly. The detective technique cooperating with the predicting data can provide more time for control strategy. Based on the redundant kinetic energy, the system can return to stability. Thus, the Closed-loop control system is viable and effective.

\section{ACKNOWLEDGMENT}

Supported by State Grid Corporation of China, Major Projects on Planning and Operation Control of Large Scale Grid（SGCC-MPLG030-2012）

\section{REFERENCES}

[1] XIE Huan, ZHANG Bao-hui, YU Guang-liang, et al. Power systems transient stability detection theory based on characteristic concave or convex of trajectory[J]. Proceedings of the CSEE, 2006, 26(5): 38-42.

[2] Xie Huan, Zhang Bao-hui. Power system transient stability detection based on characteristic concave or convex of trajectory[C]. IEEE/PES Transmission and Distribution Conference \& Exhibition:Asia and Pacific, Dalian, China, 2005.

[3] XIE Huan,ZHANG Baohui,HAO Zhiguo,et al. Self memoryprediction based on synchronous measurement of power systemmulti - variable $[\mathrm{J}]$. Electric Power Automation Equipment,2008,28（5）:1-5.

[4] Ernst D, Pavella M. Closed-loop transient stability emergency control[C]. Proceeding of IEEE/PES Winter Meeting. 2000, 1: 58-62.

[5] XIE Huan, ZHANG Baohui, YU Guangliang,et al. Transient instabilitydetection based on trajectory geometrical characteristic [J] . Proceedings of the CSEE,2008,28 (4) : :16-22.

[6] E.P.de Souzaand A.L.da Silva "An ef fi cient methodology forcoherency-based dynamicequivalents [powersystem analysis]," Proc. Inst. Elect. Eng., Gen., Transm. Distrib. C, vol.139, no.5, pp. 371--82, Sep.1992.

[7] R. Podmore, "Identi fi cation of coherent generators for dynamic equivalents,” IEEE Trans.Power App. Syst. ,vol. PAS-97, no.4, pp.1344-1354, Jul.1978. 